\title{
Perfiles de menores policonsumidores de drogas y su relación con la conducta antisocial: orientaciones para la prevención
}

\section{Profiles of polydrug users minors and problematic behavior: Guidelines for prevention.}

Fecha de recepción: 01-02-2019

Fecha de aceptación: 19-04-2019
Víctor José Villanueva Blasco Universidad Internacional de Valencia (España)

Dalila Eslava Pérez

Universidad Internacional de Valencia (España)

Andrea Vázquez Martínez

Universidad Politécnica Salesiana (Ecuador)

Bárbara González Amado

Universidad de Santiago de Compostela(España)

\section{resumen/alsstract:}

El objetivo del estudio fue elaborar un perfil de los adolescentes policonsumidores, clasificándolos de acuerdo a la edad de inicio y a sus patrones de consumo de alcohol, tabaco, cannabis y borracheras. Asimismo, se estudió si la variable comportamiento problemático diferencia entre los conglomerados resultantes. La muestra consistió en 284 alumnos y alumnas entre 14 y 17 años de los cuales el 18.3\% referían ser policonsumidores de las tres sustancias y borracheras. Se administraron la Escala de edad de inicio al uso de drogas, el Cuestionario de frecuencia de uso de drogas y la Escala de comportamiento problemático. Los resultados del análisis clúster con la muestra de policonsumidores, arrojaron una solución de tres conglomerados. Concretamente, el clúster de menores policonsumidores de riesgo está compuesto por los sujetos que inician el consumo a edades más tempranas y que presentan una mayor frecuencia del mismo, siendo además el grupo que se relaciona con una mayor frecuencia de emisión de conductas antisociales. Se destaca la importancia de una actuación por parte de los poderes públicos para asegurar el cumplimiento de la legislación, así como para el fomento de programas preventivos escolares y familiares de tipo selectivo basados en evidencia científica.

The aim of the present study was to create a polydrug users adolescents profile, classifying them according to the age of onset and their alcohol, tobacco and cannabis consumption and drunkenness. Likewise, problematic behavior variable was studied in order to assess whether it differentiates between the clusters emerged. The sample consisted of 284 students between 14 and 17 years old of whom 18.3\% were polidrug users of the three substances and drunkenness. The Scale of Age of onset in Drug Use, the Drug Use Frequency Questionnaire and the Problematic Behavior Scale were administered. Cluster analysis results have shown a three cluster solution. Specifically, the cluster of polidrug risk minors is made up of individuals who initiate their consumption at an early stage and have a greater frequency of problematic behaviors. The study highlights the relevance of actuation from public authorities with the objective of guarantying the compliance with the legislation, as well as promoting school and familiar selective preventive programs based on scientific evidence.

\section{palabras clave/keywords:}

Policonsumo, alcohol, tabaco, cannabis, adolescente, conducta antisocial.

Polydrug use, alcohol, tobacco, cannabis, adolescent, problematic behavior. 


\section{Introducción}

En Europa, durante los últimos años, el consumo de alcohol y tabaco ha sufrido un pequeño descenso, en tanto que el consumo de cannabis aumenta de forma preocupante año tras año (European Monitoring Centre for Drugs and Drug Addiction [EMCDDA], 2017).

En España, de acuerdo con la encuesta ESTUDES 2016-2017 (Plan Nacional Sobre Drogas [PNSD], 2018), realizada con estudiantes entre 14 y 18 años, sitúa la edad media de inicio del consumo de alcohol en 14 años, 14.1 años para tabaco y 14.8 años para el cannabis. La edad media de inicio de uso semanal de alcohol es de 15.1 años y para el consumo diario de tabaco es de 14.6 años. Asimismo, la prevalencia para el consumo de alcohol alguna vez en la vida se sitúa en el $76.9 \%$, un $38.5 \%$ para tabaco y un $31.1 \%$ para cannabis. Respecto a la prevalencia de consumo para los últimos 12 meses, se sitúa en el 75.6\% para el alcohol, el $34.7 \%$ para tabaco y un $26.3 \%$ para cannabis. Por su parte, la prevalencia de consumo para los últimos 30 días es del $67 \%$ para el alcohol, el $27.3 \%$ para tabaco y el $18.3 \%$ para cannabis. Asimismo, durante los últimos 30 días un $8.8 \%$ fumó tabaco diariamente, y en el caso del cannabis el $2.7 \%$ de los hombres y el $1.3 \%$ de las mujeres consumió 20 o más días en este periodo de tiempo. Respecto a las borracheras, la edad media de la primera se sitúa en los 14.7 años, un $48.9 \%$ alguna vez en la vida la ha experimentado, un $42.4 \%$ en los últimos 12 meses y un $21.8 \%$ en los últimos 30 días. En relación al policonsumo (consumo de diferentes sustancias psicoactivas en un mismo periodo de tiempo), en los últimos 12 meses un $41.6 \%$ de los encuestados consumieron dos o más sustancias (dos sustancias un 18.1\%, tres sustancias un $17.5 \%$ y cuatro sustancias o más un $17.5 \%$ ) frente a un $36.7 \%$ que solo consumió una sustancia. Respecto a las sustancias habituales que se encuentran dentro del policonsumo, el alcohol está presente generalmente en todos los consumidores de otras sustancias, concretamente, en el $97 \%$ de los que consumieron tabaco en los últimos 12 meses; así como en el $97.7 \%$ de los consumidores de cannabis. Además, el $82.5 \%$ de los consumidores de cannabis también consumieron tabaco. El $49.5 \%$ de consumidores de tabaco lo consumieron con dos sustancias más frente al $2 \%$ que sólo consumió tabaco, así como un $61.6 \%$ consumió cannabis con dos drogas más frente al $1.2 \%$ que sólo consumió cannabis.

Diversos estudios han investigado la relación entre la conducta antisocial con el alcohol (Díaz y Jiménez, 2018; Goldstein et al., 2017), con el tabaco (Weiss, Nguyen, Trung, Ngo y Lau, 2018), con el cannabis (Liu y Petras, 2016), o con el consumo de estas tres sustancias (Mason et al., 2017; Tielbeek et al., 2018). Entre los principales hallazgos de estos estudios, cabe mencionar que no encuentran relación entre el consumo estándar de alcohol y la conducta antisocial (Mason et al., 2017), pero sí en consumos problemáticos (Goldstein et al., 2017). Este hecho puede ser explicado en base a que el alcohol es la sustancia de uso más extendido, con especial carácter social (Mason et al., 2017). Aunque la conducta agresiva en una edad temprana es predictor de una conducta antisocial posterior, así como del consumo futuro de cannabis, no existe una relación causal con base empírica entre la conducta antisocial y el consumo de cannabis (Liu y Petras, 2016).

Por su parte, Liu y Petras (2016) señalan que el consumo de cannabis puede actuar como una estrategia para disminuir la alteración psicofisiológica asociada al comportamiento pro- 
blema; o bien, que los consumidores de cannabis puedan presentar un daño en su capacidad para procesar estímulos complejos, lo que les lleva a reaccionar de manera indebida. Aunque en menor medida, esta misma relación se observa en otros estudios con el consumo de alcohol y tabaco (Matuszka, Bácsai, Czobor y Gerevich, 2016), señalándose que el consumo de alcohol y tabaco interviene en la regulación del estado emocional, lo que empeora los comportamientos problemáticos.

En base a todo lo anterior, es importante intervenir desde edades tempranas en el binomio consumo de drogas y conducta antisocial, ya que el impacto negativo que puede tener sobre la trayectoria de vida de los adolescentes es destacado, tanto a nivel de salud, como social y académicamente. Además, es en la adolescencia cuando la conducta antisocial se manifiesta en mayor medida (Rodríguez, Ovejero, Bringas y Moral, 2016; Wijnen, Harakeh, Dijstra, Veenstra y Vollebergh, 2018) y aunque posteriormente a esta desciende (Moffit, 2018), si persiste hasta la edad adulta puede desembocar en un trastorno de conducta antisocial (Goldstein et al., 2017).

En consecuencia, es clave ampliar el conocimiento sobre la población de adolescentes que presentan consumos problemáticos de drogas y su relación con los comportamientos problemáticos como antecedentes de la conducta antisocial.

El objetivo del presente estudio fue elaborar un perfil de los diferentes adolescentes policonsumidores y clasificarlos de acuerdo a sus patrones de consumo de alcohol, tabaco, cannabis y la prevalencia de borracheras; así como a partir de las edades de inicio de uso de dichas sustancias. Asimismo, se estudió si la variable comportamiento problemático diferencia entre los conglomerados resultantes. Con ello se pretende orientar sobre características específicas de la población adolescente policonsumidora de estas sustancias, que permitan el diseño y/o ajuste de programas preventivos de tipo selectivo o indicado.

\section{Método}

\section{Diseño del Estudio}

Se diseñó un estudio observacional descriptivo, realizado en un contexto natural en centros educativos de Enseñanza Secundaria en España en menores de 14 a 17 años, principalmente.

\section{Participantes}

Se llevó a cabo un muestreo probabilístico por conglomerados, donde cada centro de E.S.O. público de Teruel (España) se correspondía con un conglomerado. De los tres centros participantes, se seleccionaron todos los alumnos y alumnas que cursaban $3^{\circ}$ y $4^{\circ}$ de E.S.O.

La muestra final consistió en 284 alumnos y alumnas, de los cuales el $43.7 \%$ eran varones $(\mathrm{n}=124)$ y el $56.3 \%$ mujeres $(\mathrm{n}=160)$, con una edad media de $M=15.23(D E=0.808)$. Del total de la muestra, 52 (18.3\%) referían ser policonsumidores de las tres sustancias y borracheras al menos una vez en la vida. La submuestra estaba equilibrada por sexo (27 varones vs. 25 mujeres), y las edades oscilaban entre los 15 y 17 años $(M=15.63, D E=$ $0.768)$. 


\section{Instrumentos}

Cuestionario sociodemográfico. Elaborado ad hoc, incorpora datos sobre centro escolar, unidad muestral de referencia y clave alfanumérica confidencial asignada, sexo, edad y etnicidad.

Escala de edad de inicio al uso de drogas (Agencia Antidroga de Madrid y OEDYT, 2003). En las instrucciones se solicita a los participantes que señalen la edad a la que consumieron por primera vez alcohol, tabaco, cánnabis y experimentaron su primera borrachera. La respuesta es de tipo Likert con siete opciones: Nunca (0); Once años o menos (1); A los 12 años (2); A los 13 años (3); A los 14 años (4); A los 15 años (5); y A los 16 años (6).

Cuestionario de frecuencia de uso de drogas. El cuestionario de frecuencia de uso de drogas utilizado ha sido elaborado ad hoc a partir de la encuesta ESTUDES 2012 (PNSD, 2014). Este cuestionario incorpora diversas escalas para evaluar la frecuencia de uso de tabaco, alcohol, cannabis e intoxicaciones etílicas agudas (borracheras), en distintos periodos temporales.

Para evaluar la frecuencia de uso de tabaco en los últimos 30 días, se utilizó una pregunta con formato de alternativa múltiple con cuatro opciones: Nunca (0); Menos de una vez a la semana (1); Alguna vez a la semana, pero no diariamente (2); y Diariamente (3). Una mayor puntuación es un indicador de mayor frecuencia de uso de tabaco.

La frecuencia de uso de alcohol en los últimos 12 meses se midió con una pregunta de ocho opciones de respuesta: Ningún día (0); Un día (1); Dos días (2); Tres días (3); Cuatro o cinco días (4); De seis a nueve días (5); De diez a diecinueve días (6); Veinte o más días (7). Una mayor puntuación es un indicador de mayor frecuencia de uso de alcohol.

La evaluación de la frecuencia de experimentación de intoxicaciones etílicas agudas (borracheras) en los últimos 12 meses se hizo con una pregunta abierta solicitando el número de veces. Una mayor puntuación es un indicador de mayor frecuencia de experimentación de borracheras.

Se evaluó la frecuencia de uso de cannabis en los últimos 12 meses con una pregunta de seis opciones de respuesta: Ningún día (0); Uno o dos días (1); De tres a cinco días (2); De seis a nueve días (3); De diez a diecinueve días (4); Veinte o más días (5). Una mayor puntuación es un indicador de mayor frecuencia de uso de cannabis.

Escala de comportamiento problemático (Agencia Antidroga de Madrid y OEDYT, 2003). La escala se compone de 8 ítems: a) Mentir a un profesor; b) Mentir a uno de tus padres; c) Dañar a propósito los bienes de otras personas; d) Robar cosas de una tienda; e) Golpear a alguien durante una pelea; f) Saltarse clases en el colegio; g) Copiar en el colegio; y h) Coger dinero que no era tuyo. En las instrucciones se solicita a los participantes que señalen la respuesta que consideren que mejor se ajusta a la frecuencia con la que en los últimos 12 meses han hecho alguna de las conductas expresadas en los enunciados. La respuesta es de tipo Likert con cinco opciones según el grado de acuerdo con las afirmaciones expresadas: Nunca (1); Una o dos veces (2); De tres a seis veces (3); De siete a diez veces (4); y 
Más de diez veces (5). El rango de puntuación de la escala es de 8 a 40 puntos, considerándose que una mayor puntuación es un indicador de mayor comportamiento problemático.

\section{Procedimiento}

Previamente a la administración de los cuestionarios, se facilitó al centro educativo una carta informativa con la solicitud de consentimiento informado para los tutores legales del alumnado. En ella se informaba de la voluntariedad de la participación de los menores y de la confidencialidad de los datos a través de una clave alfanumérica.

Asimismo, se protocoliza la administración de la batería de instrumentos. Al inicio se solicita al docente la confirmación de las autorizaciones de los tutores legales. A continuación, se realiza una presentación ante el alumnado explicando brevemente la investigación y solicitando su colaboración. La duración estimada es de 30 a 40 minutos, realizándose en el aula ordinaria en horario lectivo, bajo supervisión del investigador.

Para la participación en este estudio se respetaron los estándares éticos establecidos en la Declaración de Helsinki (Asociación Médica Mundial, 2013) y de la Ley de Protección de Datos española 15/1999: voluntariedad de las personas participantes, proporcionando consentimiento libre e informado para la utilización de sus datos con fines de investigación, indicándoles que podrían poner fin a su participación en el estudio en cualquier momento. Los datos fueron tratados confidencialmente respetando la intimidad de los participantes. El estudio fue aprobado por el Comité Ético de la Universidad de Santiago de Compostela (España) y el Comité Ético de Investigación Clínica de Aragón (CEICA) (España)

\section{Análisis de Datos}

Se analizaron las frecuencias de consumo en los últimos 12 meses (alcohol, borracheras y cannabis) y en los últimos 30 días (tabaco) y la edad media de inicio de consumo (alcohol, borracheras, cannabis y tabaco) en la muestra global de consumidores $(N=284)$ y en la de policonsumidores de las tres sustancias y borracheras $(n=52)$.

Previamente al análisis clúster, realizado sobre la muestra de policonsumidores, se procedió a estudiar la multicolinealidad entre las variables (i.e., frecuencia de consumo de alcohol, cannabis y borracheras en los últimos 12 meses, frecuencia de consumo de tabaco en los últimos 30 días y edad media de inicio de consumo en el alcohol, tabaco, cannabis y borracheras), a través de correlaciones bivariadas, con el fin de estudiar la independencia entre ellas. Las variables mostraron ser independientes $(r<.80)$. A continuación, se llevó a cabo un análisis de conglomerados jerárquico a partir del método de vinculación intergrupos. Este análisis preliminar ha permitido hacer una aproximación, a partir del estudio del dendograma, al número de conglomerados más adecuado. Se siguió con el procedimiento K-medias, como método de optimización, partiendo de la solución de conglomerados que se derivó de la técnica de vinculación intergrupos. Si bien los casos atípicos pueden influir en la solución óptima de conglomeración, se decidió no proceder a su eliminación puesto que dichos casos podrían formar un conglomerado determinado, lo que podría arrojar luz sobre las características diferenciales entre clústeres. 
Finalmente, con el fin de conocer cómo respondían los clústeres obtenidos ante la variable comportamiento problemático, se realizaron una serie de contrastes de medias ANOVA de un factor. Puesto que las variables no se distribuían normalmente en los tres clústeres y los tamaños de las sub-muestras eran pequeños, se utilizó la prueba no paramétrica $H$ de Kruskal-Wallis para el análisis de las diferencias entre los tres conglomerados en la dimensión comportamiento problemático, así como los ítems que la componen. Para la comparación por pares o contrastes a posteriori, se procedió con la prueba $U$ de Mann-Whitney, con corrección de Bonferroni (0.05/3), tomando .017 como el valor alpha para establecer diferencias estadísticamente significativas entre los grupos.

\section{Resultados}

\section{Descriptivos}

Las edades de inicio de consumo de cannabis son similares en la muestra global y en la de policonsumidores, pero para tabaco, alcohol y borracheras es más precoz en policonsumidores, aproximadamente medio año (ver Tabla 1).

En relación con la frecuencia de uso de las diferentes drogas, el 22.9\% de la muestra global refiere haber consumido cannabis en los últimos 12 meses, y el 5.3\% haber consumido 20

Tabla 1.- Edades de inicio de consumo de cannabis, tabaco y alcohol y de borracheras.

\begin{tabular}{|c|c|c|c|c|c|}
\hline \multirow[t]{2}{*}{ Variables } & \multicolumn{2}{|c|}{$\begin{array}{l}\text { Muestra total } \\
\qquad(N=284)\end{array}$} & \multicolumn{3}{|c|}{$\begin{array}{l}\text { Policonsumidores } \\
\qquad(N=52)\end{array}$} \\
\hline & $n(\%)$ & $M$ & $D E$ & $M$ & $D E$ \\
\hline Edad inicio alcohol & $283(99.65)$ & 12.54 & 1.16 & 12.00 & 1.20 \\
\hline Edad inicio borracheras & $179(63.03)$ & 13.52 & 1.12 & 13.11 & 1.21 \\
\hline Edad inicio tabaco & $145(51.06)$ & 13.07 & 1.39 & 12.58 & 1.40 \\
\hline Edad inicio cannabis & $55(19.37)$ & 13.31 & 1.24 & 13.42 & 1.18 \\
\hline
\end{tabular}

días o más; frente al $80.8 \%$ y al $26.9 \%$ de los policonsumidores que lo han consumido en los últimos 12 meses y que lo han usado 20 días o más, respectivamente. En cuanto al consumo de alcohol en ese mismo tramo temporal, el 35.2\% del alumnado ha tomado bebidas alcohólicas 20 días o más y un 5.3\% indican haberse emborrachado 20 días o más frente al 44\% que refieren no haber sufrido intoxicaciones etílicas. En la muestra de policonsumidores, más de la mitad (59.6\%) han tomado bebidas alcohólicas y un $15.4 \%$ se han emborrachado 
en los últimos 12 meses. Finalmente, el $18.7 \%$ de la muestra total y el $65.4 \%$ de los policonsumidores, fuman tabaco diariamente.

\section{Análisis Clúster}

El análisis de conglomerados jerárquico se llevó a cabo sobre la submuestra de policonsumidores $(n=52)$ que constituyeron el $18.31 \%$ de la muestra total. Dicho análisis arrojó una solución de 3 conglomerados: el clúster 1 estaría formado por el $63.5 \%$ de la muestra $(n=$ $33)$, el clúster 2 por el $15.4 \%(n=8)$ y el clúster 3 por el $21.2 \%(n=11)$. A partir del método de optimización, algunos de los sujetos del clúster 1 fueron reasignados al tres, quedando la distribución del siguiente modo: clúster 1 formado por el 53.8\% de la muestra $(n=28)$, el clúster 2 por el $15.4 \%(n=8)$ y el clúster 3 por el $30.8 \%(n=16)$. Los conglomerados resultantes se encontraban equiparados por sexo, $\chi^{2}(2)=3.170, p=.205$; y edad, $F(2,49)$ $=2.811 p=.70$.

El clúster 1 es el más numeroso y estaría formado por los sujetos que comienzan más tarde a consumir alcohol (12.21 años) y a emborracharse (13.36 años). No obstante, se inician antes en el consumo de tabaco (12.36 años) y en un puesto intermedio entre los otros clústeres en el consumo de cannabis o hachís (13.25 años). En cuanto a las frecuencias de consumo en los últimos 12 meses, es el grupo que menos alcohol refiere consumir (entre 10 y 19 días), menos borracheras (una media de 2 veces), y menos uso de cannabis (entre 3 y 5 días). En cuanto al consumo de tabaco en los últimos 30 días, se equiparan en frecuencia al clúster 3 , refiriendo haber fumado de media alguna vez a la semana.

El clúster 2 es el más pequeño, compuesto por aquellos sujetos que se inician a edades más precoces en el consumo de cannabis (13.13 años), alcohol (11 años) y borracheras (12.38 años); mientras que en el tabaco se sitúan en los 12.63 años, punto intermedio entre los restantes clústeres. Asimismo, refieren fumar con más frecuencia en los últimos 30 días (diariamente), y presentan frecuencias de consumo en los últimos 12 meses más elevadas para cannabis (entre 10 y 19 días) y borracheras (20 o más veces). En cuanto al consumo de alcohol, indican consumir 20 días o más en los últimos 12 meses, equiparándose al consumo de los participantes del clúster 3 .

Finalmente, el clúster 3 constituye el intermedio en tamaño, y se compone de aquellos menores que refieren iniciarse a edades más tardías que los demás en el consumo de tabaco (12.94 años) y de cannabis (13.88 años), mientras que el inicio del consumo de alcohol (12.13 años) y de las borracheras (13.06 años) se sitúan en medio de los otros clústeres. Asimismo, se equipara en frecuencia de consumo de tabaco en los últimos 30 días al clúster 1 (alguna vez a la semana) y de alcohol en los últimos 12 meses al clúster 2 (20 días o más). Por otra parte, la frecuencia de consumo en los últimos 12 meses de cannabis y de borracheras se sitúa en el medio de los clústeres 1 y 2 (entre 6 y 9 días y 7 veces, respectivamente).

\section{ANOVA de un factor}

\section{Dimensión Comportamiento problemático}

El análisis de las pruebas de normalidad y homocedasticidad en la variable comportamiento problemático, indican que los datos no se distribuyen normalmente entre los tres grupos y que sus varianzas no son homogéneas, tanto en el total de la variable como tomando aisla- 
Tabla 2.- $H$ de Kruskall-Wallis para los tres conglomerados en la variable comportamiento problemático

\begin{tabular}{|c|c|c|c|c|}
\hline Comportamiento problemático & Clúster & Rango & $H$ & $p$ \\
\hline \multirow[t]{3}{*}{ Mentir a un profesor } & 1 & 24.46 & 2.010 & .366 \\
\hline & 2 & 32.75 & & \\
\hline & 3 & 26.94 & & \\
\hline \multirow[t]{3}{*}{ Mentir a uno de tus padres } & 1 & 24.82 & 1.609 & .447 \\
\hline & 2 & 32.31 & & \\
\hline & 3 & 26.53 & & \\
\hline \multirow[t]{3}{*}{ Dañar a propósito bienes ajenos } & 1 & 26.23 & 8.188 & .017 \\
\hline & 2 & 37.38 & & \\
\hline & 3 & 21.53 & & \\
\hline \multirow[t]{3}{*}{ Robar cosas en una tienda } & 1 & 22.32 & 6.453 & .040 \\
\hline & 2 & 34.56 & & \\
\hline & 3 & 29.78 & & \\
\hline \multirow[t]{3}{*}{ Golpear a alguien durante una pelea } & 1 & 24.80 & 3.308 & .191 \\
\hline & 2 & 33.88 & & \\
\hline & 3 & 25.78 & & \\
\hline \multirow[t]{3}{*}{ Saltarse las clases en el colegio } & 1 & 22.48 & 4.808 & .090 \\
\hline & 2 & 31.56 & & \\
\hline & 3 & 31.00 & & \\
\hline \multirow[t]{3}{*}{ Copiar en el colegio } & 1 & 23.79 & 2.809 & .245 \\
\hline & 2 & 33.25 & & \\
\hline & 3 & 27.78 & & \\
\hline \multirow[t]{3}{*}{ Coger dinero que no era suyo } & 1 & 24.00 & 2.714 & .257 \\
\hline & 2 & 32.69 & & \\
\hline & 3 & 27.78 & & \\
\hline
\end{tabular}

Nota. $g l=2$ 
damente cada uno de los ítems que componen dicha variable. Por tanto, teniendo en cuenta el no cumplimiento de ambos supuestos y que los tamaños de las submuestras son pequeños, se aconseja utilizar pruebas no paramétricas.

Los resultados revelaron que no hay diferencias entre los grupos en la variable comportamiento problemático, $H(2)=4.236, p=.120$. Por otro lado, tomando los ítems de manera individualizada, los resultados mostraron diferencias estadísticamente significativas entre los sujetos de los 3 clústeres en las conductas problema "dañar a propósito los bienes de otra persona" y "robar cosas de una tienda" (ver Tabla 2).

Los contrastes a posteriori indicaron que las diferencias en la conducta de dañar a propósito bienes ajenos se concretan entre el clúster 2 y $3, U=23.500, p=.004$, siendo el rango promedio significativamente superior en el clúster $2(M=17.56)$ que en el clúster $3(M$ $=9.97$ ). No obstante, los contrastes por pares en la variable robar cosas en una tienda, no revelaron diferencias estadísticamente significativas entre ninguno de los grupos $(p>.017)$.

\section{Discusión}

En esta investigación se ha analizado la edad de inicio al consumo de alcohol y borracheras, tabaco y cannabis. Las edades medias de inicio halladas son para todos los casos inferiores a las reportadas por la encuesta ESTUDES (PNSD, 2018), situándose entre un año y un año y medio más precoces. Por su parte, en la submuestra de policonsumidores, las edades medias observadas son para alcohol dos años inferior a la encuesta ESTUDES y medio año respecto a la muestra total; para borracheras algo más de un año y medio inferior a la encuesta ESTUDES, y próximo a medio año inferior a la muestra total; para tabaco un año inferior a la encuesta ESTUDES, y aproximadamente medio año inferior a la muestra total; y para cannabis un año y medio inferior a la encuesta ESTUDES, y prácticamente similar a la muestra total.

Los datos sobre edades de inicio halladas en este estudio pueden explicarse parcialmente al considerar las relativas a la Comunidad Autónoma de Aragón (a donde corresponde el municipio de Teruel) que reporta la encuesta ESTUDES (PNSD, 2018), siendo de 13.5 años para el alcohol, 13.6 años para tabaco y 14.6 años para cannabis, inferiores a la media nacional. De esta observación se deriva la necesidad de analizar conjuntamente los patrones de consumo de drogas en población adolescente y la presencia de factores de riesgo y protección que pueden ser específicos según las características sociodemográficas y culturales. En este caso, los procesos de influencia social directa (presión) (p.e. McDonough, Jose y Stuart, 2016) o indirecta (expectativas grupales, modelado, etc.) (p.e. Gazis, Connor y Ho, 2010) pueden ser más relevantes en entornos de predominancia rural frente a urbana donde suele haber una menor oferta de alternativas de ocio específicas según edad y los espacios de socialización suelen ser comunes intergeneraciones (Moreta-Herrera, Mayorga-Lascano, León-Tamayo e Ilaja-Verdesoto, 2018).

Respecto a los hallazgos relativos a la submuestra de menores policonsumidores, al aplicar el análisis clúster se observan tres clústeres que presentan patrones diferentes de frecuencia de consumo de drogas y edades de inicio en el consumo, siendo estas inferiores a las de la 
muestra total. A continuación se presentan las denominaciones que se establecieron de estos clústeres en base al nivel de riesgo que se asocia a la frecuencia de consumo de las distintas sustancias:

- Clúster 1, menores policonsumidores de riesgo leve: respecto a otros menores policonsumidores, presentan edad de inicio en el consumo inferior a la media para tabaco y superior para cannabis, alcohol y borracheras, y una menor frecuencia de consumo para estas sustancias.

- Clúster 3, menores policonsumidores de riesgo moderado: respecto al clúster de menores policonsumidores de riesgo leve, presentan una edad de inicio en el consumo inferior para alcohol y borracheras, y mayor frecuencia de consumo para cannabis, alcohol y borracheras.

- Clúster 2, menores policonsumidores de riesgo elevado: respecto a otros menores policonsumidores (leves o moderados), muestran una edad de inicio en el consumo inferior a la media para cannabis, alcohol y borracheras, y mayor frecuencia de consumo para estas sustancias y tabaco.

Estos datos señalan que, en términos generales, aquellos adolescentes que se inician en el consumo de estas drogas a edades más tempranas presentan un patrón de mayor frecuencia que los que se inician a edades posteriores. A este respecto, hay amplio consenso científico en que el inicio temprano en el consumo de drogas, específicamente antes de los 15 años, es un importante factor de riesgo para el consumo futuro de drogas (Caneto, del Valle Vera, Pautassi y Pilatti, 2015). Concretamente, un inicio temprano en el consumo de drogas legales (alcohol y tabaco) aumenta la probabilidad de inicio en el consumo de drogas ilegales (Becoña et al., 2011; Sánchez-Niubò, Sordo, Barrio, Idave y Domingo-Salvany, 2018; Sloboda, 2008), especialmente cannabis (Becoña, 2002; Villanueva, 2017). Esto concuerda con nuestros hallazgos. Si atendemos a la prevalencia de consumo de cannabis en la muestra total y en la de policonsumidores, se observa que siendo estos últimos 52 participantes, representan el $94.5 \%$ de aquellos de la muestra total que habían consumido cannabis en los últimos 12 meses.

En definitiva, una edad de inicio precoz en el consumo de alcohol aumenta la probabilidad de iniciarse tempranamente en el consumo abusivo de esta sustancia (borracheras) y de cannabis, así como presentar una mayor frecuencia de consumo de estas sustancias. Esta relación se observa igualmente con una edad de inicio precoz en el consumo de tabaco, aunque en menor medida. En consecuencia, aunque es importante el abordaje preventivo de cualquier sustancia, el de las drogas legales es prioritario dado que estos contactos abren la puerta a la progresión hacia consumos abusivos y consumos de otras sustancias (Kandel, 1980; Sánchez-Niubò et al., 2018).

Respecto a la relación del consumo de drogas con el comportamiento problemático, los resultados del presente estudio indican que los menores policonsumidores de riesgo elevado (clúster 2), que refieren una mayor frecuencia de uso de drogas, también son los que más comportamientos problemáticos realizan, seguidos a este respecto por los policonsumidores de riesgo moderado (clúster 3). Entre estas conductas destacan por ser estadísticamente 
significativas la de dañar a propósito bienes ajenos y robar cosas en una tienda. Este hallazgo está en consonancia con otros estudios que indican que los adolescentes que presentan mayores consumos de drogas muestran una probabilidad mayor de realizar actividades predelictivas, como el daño deliberado a bienes públicos o el robo de artículos (Calafat et al., 2000; Pérez y Ruiz, 2017). Asimismo, tal y como señalan algunos autores (Liu y Petras, 2016; Mashhoon, Betts, Farmer y Lukas, 2018; Matuszka et al., 2016; Modecki, ZimmerGembeck y Guerra, 2017) el consumo de alcohol, tabaco y/o cannabis puede afectar al funcionamiento de diversos sistemas cognoscitivos, entre ellos los implicados en la toma de decisiones, favoreciendo la conducta antisocial.

Son diversos los estudios que señalan cómo el consumo de drogas puede perjudicar el proceso de maduración de diversas estructuras cerebrales, o a diversas funciones cognoscitivas, generando un deterioro temporal o permanente (Montoya-Filardi y Mazón, 2017; NguyenLouie et al., 2017). Y aunque esta relación puede ser bidireccional, es evidente la conveniencia de retrasar la edad de inicio del consumo de sustancias, legales o ilegales.

A partir de todo lo anterior, cabe destacar que las elevadas prevalencias de menores adolescentes consumidores y policonsumidores de alcohol, tabaco y cannabis reportadas, dan idea de la exposición, esporádica o continuada, de estos menores a los riesgos asociados al consumo de drogas que pueden afectar a su desarrollo evolutivo. De igual modo, constatan el grado de accesibilidad y normalización de su consumo.

Son diversas las acciones que pueden implementarse, desde una perspectiva integral, tanto en el área de reducción de la oferta como de la demanda, así como en los ámbitos comunitario, familiar y escolar. Entre algunas de estas acciones orientadas a disminuir la probabilidad de que los menores se inicien y progresen en el consumo de drogas, y algunos desarrollen perfiles de policonsumo de menor o mayor gravedad, destacaríamos:

1 En el área de reducción de la oferta, si bien la lucha contra el narcotráfico forma parte indispensable dentro de este marco de actuación, las acciones para el efectivo cumplimiento de la legislación vigente en materia de venta y acceso de drogas legales es igualmente, o más, relevante. Por tanto, abogamos por una mayor cobertura y compromiso en la aplicación de la legislación, especialmente cuando la oferta va orientada a menores, y aspectos como la publicidad encubierta y el afán lucrativo vulneran y ponen en riesgo su salud y desarrollo psicoevolutivo. Medidas tales como el aumento de las sanciones administrativas y la aplicación de medidas judiciales de responsabilidad civil ante el incumplimiento de la legislación se encuadrarían en esta área.

2 En el área de reducción de la demanda, conviene invocar la Ley Orgánica 1/1996 de Protección Jurídica del Menor. Aunque esta no habla concretamente de la protección del menor frente a las drogas, en su artículo 17 establece como un derecho que le asiste su protección frente a cualquier situación que perjudique su desarrollo personal o social, correspondiendo a los poderes públicos garantizar dicho derecho. En base a este artículo, se propone: 
- Desvelar la proliferación de actuaciones pseudo-preventivas (en algunos casos con posibles efectos iatrogénicos), promoviendo una regulación de la prevención a través de procesos de acreditación de programas y profesionales, y supeditando el acceso a la financiación pública al cumplimiento de criterios básicos de calidad, como los impulsados por el Observatorio Europeo sobre Drogas y Toxicomanías (2013).

- Promover este tipo de programas especialmente en la etapa de transición entre la educación Primaria y Secundaria, ya que la literatura científica (Robertson et al., 2004) y los hallazgos presentados señalan que es una etapa crítica, adelantando la implementación de los programas orientados a las drogas legales a la última etapa de Primaria, con especial énfasis en la prevención del alcohol.

- Al hablar de menores policonsumidores, desarrollar elementos de screening del inicio precoz en el consumo de drogas, orientados tanto al ámbito familiar como educativo y Atención Primaria, que permitan la detección temprana y puesta en marcha de intervenciones de consejo breve y/o la inclusión en programas de prevención selectiva.

- Vinculación de las actuaciones socio-educativas, de sanción administrativa y penal en menores relacionadas con la conducta antisocial, a programas de prevención selectiva o indicada en materia de consumo de drogas.

- La inclusión de la familia en los programas de prevención selectiva. Se dispone de evidencia de que los programas de prevención selectiva que trabajan desde el ámbito familiar obtienen más éxito que los que sólo intervienen con los menores (Burkhart, 2006; Orte, Ballester, Amer y Vives, 2017).

- Regulación de los espacios familiares sin humo. Dado que los progenitores o tutores legales son modelos en relación a hábitos, actitudes y comportamiento, el uso de una sustancia por parte de uno o ambos progenitores, o una actitud favorable o condescendiente hacia las drogas, incrementa la probabilidad de uso en los hijos (Barragán et al., 2016; Becoña, 2002; Becoña et al., 2011; Sloboda y David, 1997). Una propuesta novedosa es la prohibición de fumar tabaco en un vehículo si en este viaja un menor para evitar su exposición al humo de segunda mano.

Entre las limitaciones del estudio se encuentran por un lado las relacionadas con la muestra, por su tamaño y procedencia, siendo interesante conocer los resultados de otras zonas geográficas así como contar con una muestra mayor. Por otro lado, se trata de un estudio con un diseño trasversal, no permite establecer relaciones causales entre las variables de interés para el estudio. Líneas futuras de investigación deben contribuir a profundizar en el conocimiento y orientaciones para la actuación con menores policonsumidores de drogas. En este sentido, se precisan estudios longitudinales que permitan establecer trayectorias de vulnerabilidad y riesgo previas a la situación de policonsumo; la coincidencia en otros estudios con los perfiles de policonsumidores hallados en este; y la pertinencia de aplicar la perspectiva 
de género en el análisis de las tipologías de policonsumidores, así como en relación a las trayectorias que las determinan, atendiendo a aquellos factores de riesgo comunes con los comportamientos problemáticos y la conducta antisocial, y los mapas de interacciones entre estas variables que favorecen trayectorias de vida vulnerables.

\section{Referencias}

Asociación Médica Mundial. (2013, Octubre). Declaración de Helsinki. Principios éticos para las investigaciones médicas en seres humanos. 64a. Asamblea General, Fortaleza, Brasil. Recuperado de http://www. wma.net/es/30publications/10policies/b3/

Bandura, A. (1977). Self-efficacy: toward a unifying theory of behavioral change. Psychological Review, 84, 191-215.

Barragán, A. B., Martos, Á., Simón, M. M., Pérez-Fuentes, M. C., Molero, M. M., y Gázquez, J. J. (2016). Consumo de tabaco y alcohol en adolescentes y relación con la familia. European Journal of Child Development, Education and Psychopathology, 4(1), 49-61. doi: 10.1989/ejpad.v4i1.34

Becoña, E. (2002). Bases científicas de la prevención de las drogodependencias. Ministerio del Interior. Delegación del Gobierno para el Plan Nacional sobre Drogas. Madrid: Plan Nacional sobre Drogas.

Becoña, E., Cortés, M., Arias, F., Barreiro, C., Berdullas, J., Iraurgui, I.,...Villanueva, V. J. (2011). Manual de adicciones para psicólogos especialistas en psicología clínica en formación. España: Sociedad Científica Española de Estudios sobre el Alcohol, el Alcoholismo y las otras Toxicomanías.

Calafat, A., Juan, M., Becoña, E., Ferrández, C., Gil, E., Palmer, A., Sureda, P., y Torres, M. (2000). Salir de marcha y consumo de Drogas. Madrid: Delegación del Gobierno para el Plan Nacional sobre Drogas.

Caneto, F., del Valle Vera, B., Pautassi, R. M., y Pilatti, A. (2015). Personalidad, edad de inicio y problemas por consumo de alcohol en estudiantes. Quaderns de Psicologia, 17 (2), 19-35. doi: 10.5565/rev/qpsicologia. 1247

Díaz, N., y Jiménez, M. (2018). Consumo de alcohol, conducta antisocial e impulsividad en adolescentes españoles. Acta Colombiana de Psicología, 21 (2), 110-130. doi: 10.14718/ACP.2018.21.2.6

European Monitoring Centre for Drugs and Drug Addiction. (2017). Drug treatment expenditure: A methodological overview, EMCDDA Insights 24. Luxembourg: Publications Office of the European Union.

Gazis, N., Connor, J. P., y Ho, R. (2010). Cultural identity and peer influence as predictors of substance use among culturally diverse Australian adolescents. Journal of Early Adolescence, 30 (3), 345-368. doi: 10.1177/0272431609333276

Goldstein, R. B., Chou, S. P., Saha, T. D., Smith, S. M., Jung, J., Zhang, H.,...Grant, B.F. (2017). The epidemiology of antisocial behavioral syndromes in adulthood: results from the National Epidemiological Survey on Alcohol and related conditions-III. Journal of Clinical Psychiatry, 78 (1), 90-98. doi:10.4088/JCP.15m10358

Kandel, D. B. (1980). Developmental stages in adolescent drug involvement. NIDA Research Monograph, 30, 120-127.

Ley Orgánica 1/1996, de 15 de enero, de Protección Jurídica del Menor. Boletín Oficial del Estado, núm. 15, de 17 de enero de 1996, pp. 13-24. Recuperado de https://www.boe.es/buscar/pdf/1996/BOE-A-1996-1069-consolidado.pdf

Liu, W., y Petras, H. (2016). Aggressive Behavior and Cannabis Use. NORC working paper series. Bethesda, MD, US.

Mashhoon, Y., Betts, J., Farmer, S. L., y Lukas, S. E. (2018). Early onset tobacco cigarette smokers exhibit deficits in response inhibition and sustained attention. Drugs and Alcohol Dependence, 184(1), 48-56. doi: 10.1016/j.drugalcdep.2017.11.020

Mason, M. J., Zaharakis, N. M., Rusby, J. C., Westling, E., Light, J. M., Mennis, J., y Flay, B. R. (2017). A longitudinal study predicting adolescent tobacco, alcohol and cannabis use by behavioral characteristics of close friends. Psychology of Addictive Behaviors, 31 (6), 712-720. doi: 10.1037/abd0000299

Matuszka, B., Bácskai, E., Czobor, P, y Gerevich, J. (2016). Physical Aggression and concurrent alcohol and tobacco use among adolescents. International Journal of Mental Health and Addiction, 15 (1), 90-99. doi: 10.1007/s11469015-9630-6 
McDonough, M., Jose, P. y Stuart, J. (2016). Bi-directional effects of peer relationship and adolescent substance use: a longitudinal study. Journal of Youth Adolescence, 45, 1652-1663. doi: 10.1007/s10964-015-0355-4

Modecki, K. L., Zimmer Gembeck, M. J. y Guerra, N. (2017). Emotion regulation, coping, and decision making: three linked skills for preventing externalizing problems in adolescence. Child Development, 88(2), 417-426. doi: 10.1111/ cdev.12734

Moffitt, T. (2018). Male antisocial behaviour in adolescence and beyond. Nature Human Behavior, 2, 177-186. doi: 10.1038/ s41562-018-0309-4

Montoya-Filardi, A. y Mazón, M. (2017). El cerebro adicto: imagen de las complicaciones neurológicas por el consumo de drogas. Radiología, 59 (1), 17-30. doi 10.1016/j.rx.2016.09.005

Moreta-Herrera, R., Mayorga-Lascano, M., León-Tamayo, L., y llaja-Verdesoto, B. (2018). Consumo de sustancias legales, ilegales y fármacos en adolescentes y factores de riesgo asociados a la exposición reciente. Health \& Addictions/Salud y Drogas, 18 (1), 39-50. doi: 10.21134/haaj.v18i1.333

Nguyen-Louie, T. T., Matt, G. E., Jacobus, J., Li, I., Cota, C., Castro, N. y Tapert, S. F. (2017). Earlier alcohol use onset predicts poorer neuropsychological functioning in young adults. Alcoholism: Clinical and Experimental Research, 41(12), 2082-2092. doi: 10.1111/acer.13503

Observatorio Español de la Droga y las Toxicomanías. (2018). Encuesta sobre uso de drogas en Enseñanzas Secundarias en España (ESTUDES) 2016-2017. Madrid: Ministerio de Sanidad, Servicios Sociales e Igualdad.

Observatorio Europeo sobre Drogas y Toxicomanías. (2013). Criterios de calidad europeos en prevención de drogas: una guía breve. Recuperado de http://www.emcdda.europa.eu/attachements.cfm/att_220566_ES_ Est\%C3\%A1ndares\%20prevenci\%C3\%B3n\%200EDT\%20ES\%2024.07.2014.pdf

Orte, C., Ballester, L., Amer, J. y Vives, M. (2017). Género y cambio de actitudes ante el consumo de drogas. Los efectos del Programa de Competencia Familiar con adolescentes (12-14 años). Universitas Psychologica, 16 (4), 234245. doi: 10.11144/javeriana.upsy16-4.gcac

Pérez, E., y Ruiz, S. (2017). El consumo de sustancias como factor de riesgo para la conducta delictiva: Una revisión sistemática. Acción Psicológica, 14 (2), 33-50. doi: 10.5944/ap.14.2.20748

Robertson, E., David, S. y Rao, S. (2004). Cómo prevenir el uso de drogas en los niños y los adolescentes. Una guía con base científica para padres, educadores y líderes de la comunidad (NIH Publication No. 04-4214). Recuperado de https://www.drugabuse.gov/sites/default/files/redbook_spanish.pdf

Rodríguez, F. J., Ovejero, A., Bringas, C. y Moral, M. V. (2016). Afrontamiento de conflictos en la socialización adolescente. Propuesta de un modelo. Psicología desde el Caribe, 33(1), 1-13.

Sánchez-Niubò, A., Sordo, L., Barrio, G., Indave, B. I., y Domingo-Salvany, A. (2018). Inicio y progresión en el consumo de sustancias en la población general de Cataluña, España. Adicciones. Avance online. doi: 10.20882/adicciones. 1089

Sloboda, Z. (2008). Moving towards evidence-based practice: School-based prevention of substance use in the USA. En S. Rödner, B. Olsson y R. Room (Eds.), A cannabis reader: global issues and local experiences Perspectives on cannabis controversies, treatment and regulation in Europe (pp. 247-248). Luxembourg: Office for Official Publications of the European Communities.

Sloboda, Z., y David, S. L. (1997). Preventing drug use among children and adolescents: A research-based guide. Bethesda, MD: National Institute of Mental Health.

Tielbeek, J. J., Vink, J. M., Polderman, T. J. C., Popma, A., Posthuma, D. y Verweij, K. J. H (2018). Genetic correlation of antisocial behavior with alcohol, nicotine and cannabis use. Drug and Alcohol Dependence, 187 (1), 296-299. doi: 10.1016/j..drugalcdep.2018.03.030

Villanueva, V. J. (2017) Diseño y evaluación de la eficacia de un programa de habilidades para la vida en la prevención del consumo de sustancias en adolescentes (Tesis doctoral). Universidad de Santiago de Compostela, Santiago de Compostela.

Weiss, B., Nguyen, T., Trung, L., Ngo, V. y Lau, A. (2018). Tobacco smoking and antisocial deviance among Vietnamese, Vietnamese-American, and European American adolescents. Journal of Abnormal Child Psychology, 47, 5969. doi:10.1007/s10802-018-0416-8 
Wijnen, L.L., Harakeh, Z., Dijstra, J.K., Veenstra, R. y Vollebergh, W. (2018). Aggressive and prosocial peer norms: change, stability and associations with adolescent aggressive and prosocial behavior development. Journal of Early Adolescence, 38 (2), 178-203. doi: 10.1177/0272431616665211 